\title{
Multiscale simulation of mixing processes using 3D-parallel, fluid- structure interaction techniques
}

\author{
R. Valette ${ }^{1}$, B. Vergnes ${ }^{1}$, T. Coupez ${ }^{1}$ \\ ${ }^{1}$ CEMEF - Ecole des Mines de Paris - Paristech - UMR CNRS 7635, Rue Claude Daunesse, \\ 06904 Sophia Antipolis, France \\ URL: www.cemef.ensmp.fr \\ e-mail: rudy.valette@ensmp.fr, bruno.vergnes@ensmp.fr, \\ thierry.coupez@ensmp.fr
}

\begin{abstract}
This work focuses on the development of a general finite element code, called Ximex ${ }^{(B)}$, devoted to the three-dimensional direct simulation of mixing processes of complex fluids. The code is based on a simplified fictitious domain method coupled with a "level-set" approach to represent the rigid moving boundaries, such as screws and rotors, as well as free surfaces. These techniques, combined with the use of parallel computing, allow computing the time-dependent flow of generalized Newtonian fluids in large and complex processes, involving moving free surfaces which are treated by a level-set/Hamilton-Jacobi method. Two flow case studies will be presented in this paper: the flow within a twin-screw extruder and the flow in a batch mixer.
\end{abstract}

Key words: Finite elements, Distributive and dispersive mixing, Free surface flows

\section{INTRODUCTION}

In industrial mixing processes, as twin screw compounding or batch mixing, the general purpose is to disperse a material (a filler or a fluid) into another, in order to obtain a desired morphology (size and homogeneity of the dispersed phase). This is generally achieved by applying stresses and strain in complex flow situations (unsteady conditions, free surface flows...). The development of computational techniques allows now the approach of such mixing processes by numerical simulation. Direct numerical simulation permits to access to accurate information on the flow conditions (velocity and stress field, trajectories, temperature field...) and thus to a better understanding and control of the industrial process. But difficulties to develop such numerical codes are numerous.

First of all, the absence of symmetry of the moving boundaries (the screws or the rotors) implies that their rigid body motion has to be taken into account by using a special treatment. The so-called fictitious domain method, which consists in embedding the flow domain in a larger computational domain, has already been used in previous studies $[1,2]$ to overcome this difficulty. We present here a simplified application of this method being the rigid body motion imposed by using a multiphase/penalty method $[3,4]$.

\section{PROBLEM FORMULATION, FLUID DOMAIN AND BOUNDARY CONDITIONS}

Let us consider the flow of an incompressible fluid in a domain $\Omega(t)$ representing the domain occupied by the fluid inside a portion of a twin screw extruder or a batch mixer. For a Newtonian fluid, the velocity / pressure $(\boldsymbol{u}, p)$ problem is governed by mass and momentum equations :

$\left\{\begin{array}{c}-\nabla p+2 \eta \Delta \mathbf{u}=0 \\ \nabla . \mathbf{u}=0\end{array}\right.$

No slip boundary conditions on the moving screws and on the barrel walls are imposed. Solving this problem by discretizing only the domain occupied by the fluid [5] would imply mesh distortion and dynamic remeshing. To avoid this drawback, one uses a fixed Eulerian computational domain which contains the "domain" occupied by the moving boundaries, i.e. the screws. Equations (1) are then solved in a multiphase approach in which a "one fluid" model is solved in each position $\boldsymbol{x}$ and time $t$, with different prescribed values of its viscosity 
parameter, depending on the phase (air, liquid, solid) present at $(\boldsymbol{x}, t)$. For a given value of the liquid viscosity, an air/liquid and liquid/solid ratio of $1 / 1000$ was used in the multiphase approach.

\section{FINITE ELEMENT SOLVER}

The global velocity-pressure problem is solved by using a P1+/P1-based (MINI-element) mixed finite element method, each solid sub-domain (screw, rotor) being represented by a surface mesh issuing from $\mathrm{CAD}$, and the liquid-air interface being separated by a "level-set" function [6].

The algebraic problems resulting from the finite element formulation of (1) were solved using the conjugate residual method associated to the incomplete LU preconditioner from the PETSC (Portable Extensive Toolkit for Scientific Computation) library. A master-slave parallel strategy was used [7], involving SPMD (single program, multiple data) modules and the MPI (message passing interface) library standard.

\section{MESH IMMERSION TECHNIQUE AND MULTIPHASE MODELING}

To identify the position of the screw/fluid interfaces, one uses a P1 approximation of the distance to these interfaces, as for "level set" methods. When screw / mixer geometry is taken from a surface mesh CAD file, the distance to each surface mesh element is calculated with a hierarchical method. Solid body motion is accounted for by directly assigning velocities to nodes of the grid (as for usual boundary conditions) belonging to elements located inside the screws. The treatment of boundary elements is done by using a "penalty" mixing rule on viscosities as proposed by Basset et al. [8]. More precisely, the "level set" function $\alpha(x)$ is used on each element to approximate a fill factor $I_{\Omega}$ (which is used as a weighting factor in the mixing rule) for the fluid phase as follows:

$$
I_{\Omega}=\frac{\alpha^{+}}{|\alpha|}
$$

where $\alpha+$ and $|\alpha|$ are the sum of, respectively, all positive (chosen as the "inside" domain) values and absolute values of $\alpha$. The same method is used to compute a free surface flow, using a "level set" approach for the identification of the surface position at each time step [8]. The "level set" method will perform well in the advection of scalar fields that do not present high gradients $(|\nabla \alpha| \sim 1)$. Nevertheless, the transport of the "level set" function does not guarantee that this property is automatically satisfied. One then needs to reinitialize the "level set" function periodically by substituting a smoother function $\varphi(x)$ that shares the same zero isovalue. This can be accomplished by solving a Hamilton-Jacobi equation at each time step:

$$
\frac{\partial \varphi}{\partial \theta}=S(\varphi)(1-|\nabla \varphi|)
$$

where $\theta$ is a fictitious time and $S$ is a "sign" function, the initial condition for $\varphi$ being initialized with $\varphi(x, 0)=\alpha(x, t)$.

\section{APPLICATIONS}

To illustrate the potentiality of the proposed method, we present three applications in the domain of industrial mixing. The first one concerns the flow of a molten polymer in a filled portion of twin screw extruder, the second one the dispersion of solid fillers in a polymer matrix in an internal mixer and the third one the flow of a fluid in a batch mixer, with free surface. All these results have been obtained with the commercial version of Ximex ${ }^{\circledR}$, using three $2.8 \mathrm{GHz}$ Intel bi-Xeon computers in parallel, eight $2.4 \mathrm{GHz}$ AMD Opteron computers in parallel and one $3.2 \mathrm{GHz}$ Intel Pentium IV computer for, respectively, computing the following twinscrew extrusion, internal mixer and batch mixer processes simulations.

\subsection{Twin-screw extrusion}

Fig. 1 presents a portion of intermeshing co-rotating twin screw extruder, including right- and left-handed screw elements. It is totally filled by a viscous fluid, obeying a power law behavior. Fig. 1 shows the pressure field, calculated with boundary conditions imposing the same pressure $(p=0)$ at the entry and exit planes. In this case, the flow is symmetrical and the flow rate equal to zero. It is what can be seen in the figure, with a maximum of pressure attained at 
the junction between the two elements.

Fig. 2 presents another screw profile, with a block of kneading discs in neutral configuration (staggering angle $90^{\circ}$ ) situated in between right-handed elements. In this case, the flow rate is imposed at the entry plane. The resulting pressure profile is indicated in Fig. 2. We can see that the pressure is maximum in front of the disc tips, and minimum behind the tip. Moreover, as the neutral discs cannot convey the polymer, the pressure tends to decrease along the block, as shown in the cross section above the $3 \mathrm{D}$ view.

\subsection{Internal mixer}

In the second example, we consider an internal mixer, with two counter-rotating rotors in an eightshaped chamber. The objective is to follow the distribution of 1000 solid particles injected at time $t=0$ in the space between the rotors. Fig. 3 shows the positions of these particles at different times, what allows quantifying the mixing ability of the system. From these results, we can also apply a kinetic theory to predict mass density distributions (as shown on Figure 4) [3]. Starting from a unique class of agglomerates of $50 \mu \mathrm{m}$ radius, we can follow during the mixing time the evolution of this population. By an erosion mechanism, particles of $2 \mu \mathrm{m}$ radius are extracted from the agglomerates, leading to the reduction of the agglomerates radius and the development of a new class of small particles.

\subsection{Batch mixer}

For this last example, we consider a batch mixer with two rotating anchors, as shown in Fig. 5. The bowl is also rotating, which leads to very complex flow patterns. In this case gravity forces are taken into account. Fig. 5 shows that Ximex ${ }^{\circledR}$ software is able to calculate the velocity field and to track accurately the free surface during the mixing process.

\section{CONCLUSIONS}

In this paper, we present a direct 3D simulation code for extrusion and mixing processes, called Ximex ${ }^{\circledR}$. Based on performing numerical techniques, including fictitious domains method, "level set" approaches and massive parallelization, it allows calculating the flow conditions in processes with complex geometries and kinematics, as those usually encountered in mixing operations. To illustrate the capacities of this numerical tool, we have presented different examples, for extruders and batch mixers.

\section{REFERENCES}

1. Bertrand, F., Tanguy, P. A., Thibault, F., A threedimensional fictitious domain method for incompressible fluid flow problems, Int. J. Num. Meth. Fluids, 25, 719 (1997).

2. Avalosse, T., Rubin, Y., Analysis of mixing in corotating twinscrew extruders through numerical simulation, Intern. Polym. Processing XV (2), 117 (2000).

3. Valette, R., Bruchon, J., Digonnet, H., Laure, P., Leboeuf, M., Silva, L., Vergnes, B., Coupez, T., Méca. Indus., 8, 251 (2007).

4. Del Pino, S., Pironneau, O., A fictitious domain based general PDE solver, in Numerical methods for scientific computing: variational problems and applications, Y. Kuznetsov, P. Neittanmaki, O. Pironneau eds., CIMNE, Barcelona (2003).

5. Pokriefke G., Numerical simulation of viscous flow in a partially filled co-rotating twin screw extruder, Intern. Polym. Processing XXII (1), 61 (2007).

6. Sussman, M., Smereka, P., Osher, S., J. Comp. Phys., 114, 146 (1994).

7. Digonnet, H., Coupez, T., Object-oriented programming for "fast-and-easy" development of parallel applications in forming processes simulation, in K.J. Bathe, (ed.), Second MIT Conference on Computational Fluid and Solid Mechanics (2003).

8. Basset, O., Digonnet, H., Guillard, H., Coupez, T., Proc. Int. Conf. on Comput. Methods for Coupled Problems in Science and Engineering, Barcelona (2005).

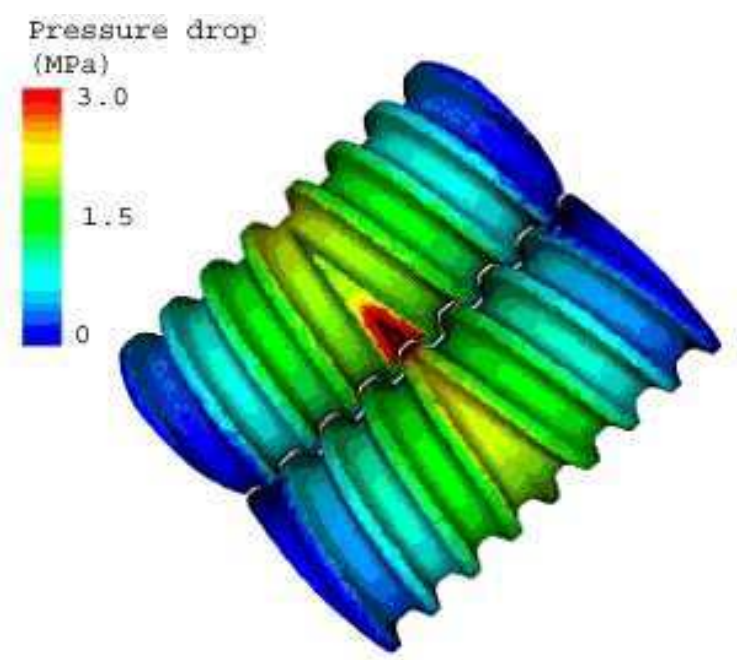

Fig. 1. Pressure field in a twin screw extruder for a combination of left- and right-handed elements. 


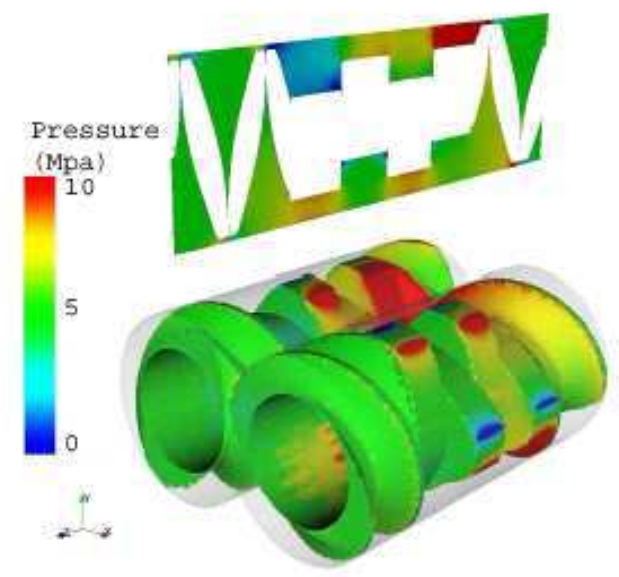

Fig. 2. 3D view and cross section of the pressure field in the computational domain of Fig. 2.

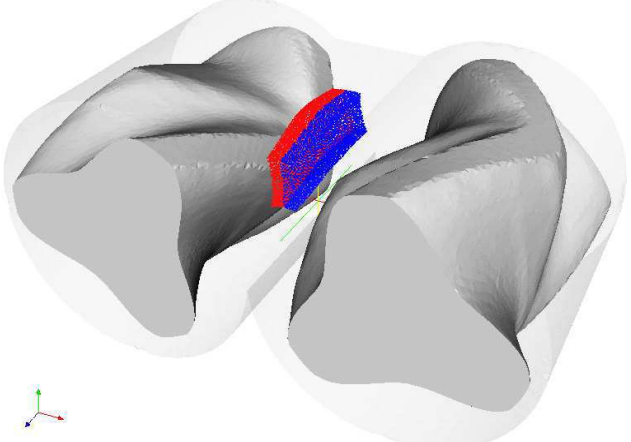

(a)

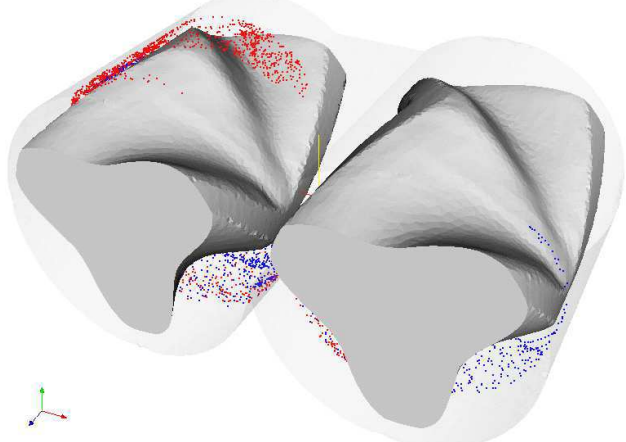

(b)

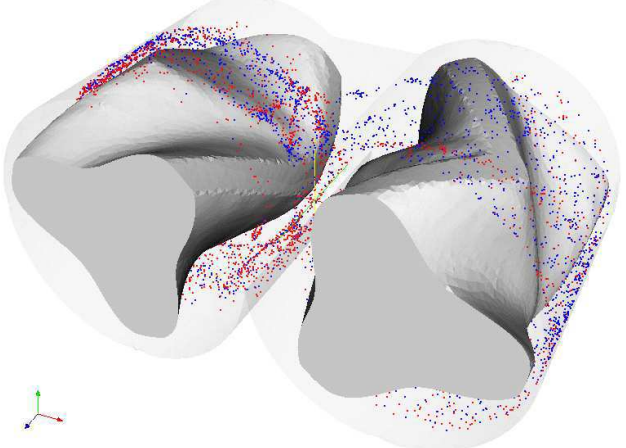

(c)

Fig. 3. Positions of particles in the internal mixer after 0 (a), 1 (b) and $3 \mathrm{~s} \mathrm{(c).}$
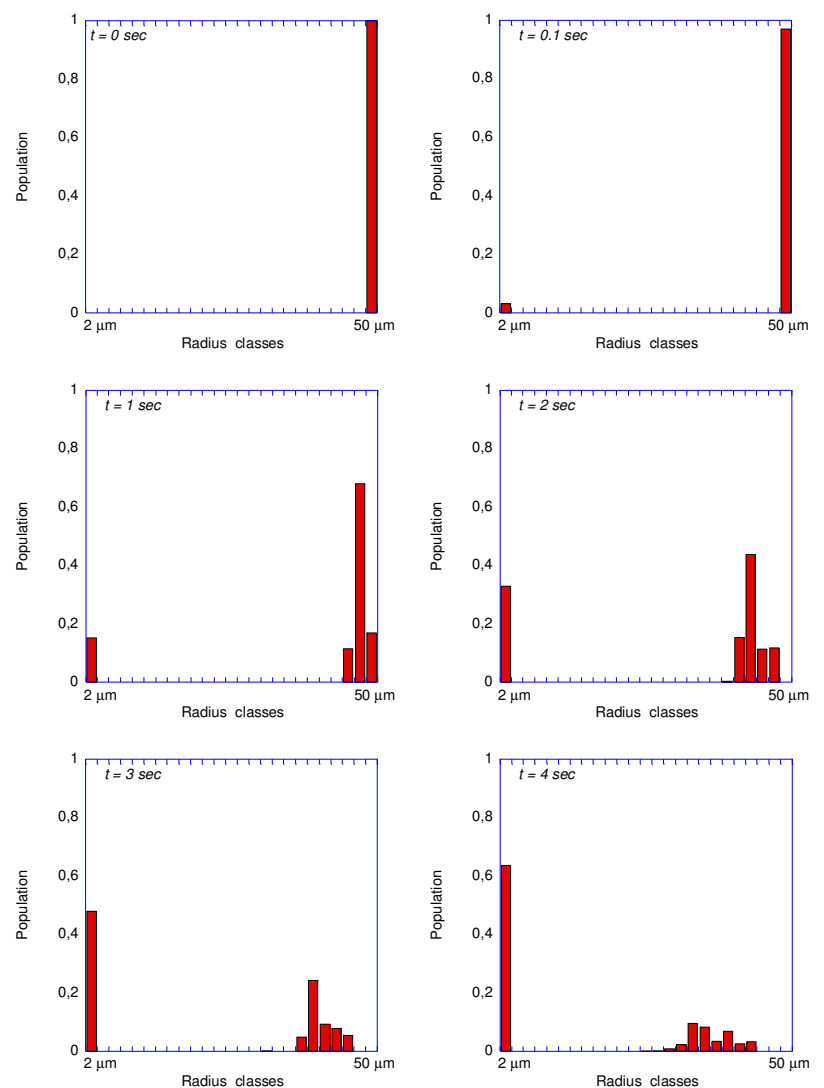

Fig. 4. Mass fraction histograms of each class of particles (radius 2 to 50 microns), at different mixing times: $\mathrm{t}=0 \mathrm{~s}$, $0.1 \mathrm{~s}, 1 \mathrm{~s}, 2 \mathrm{~s}, 3 \mathrm{~s}$ et $4 \mathrm{~s}$.

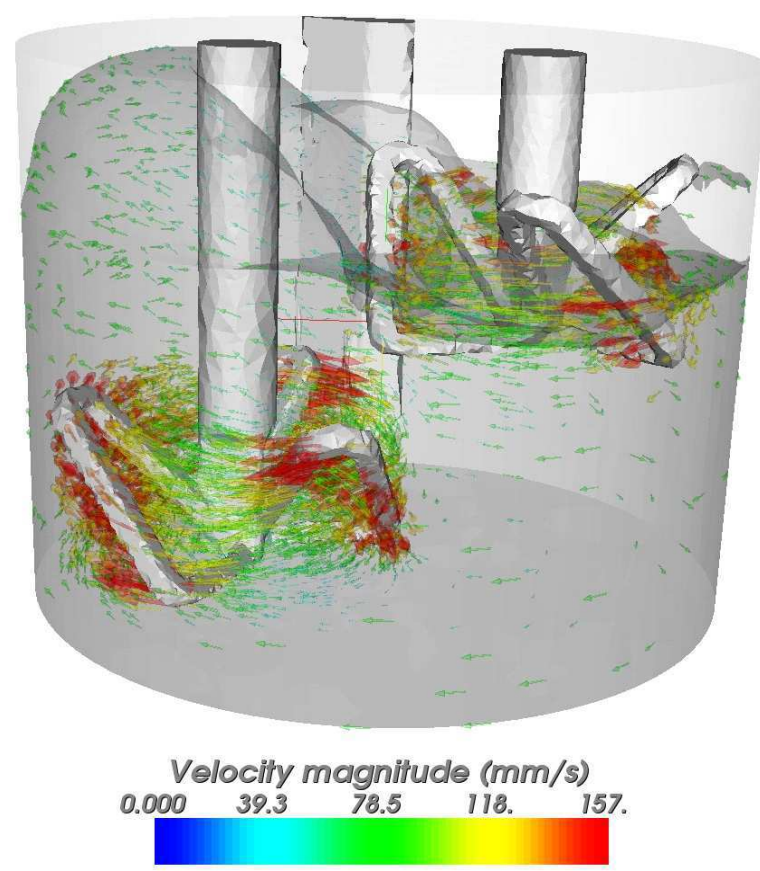

Fig. 5. Free surface position and velocity field in a batch mixer. 